\title{
The Asymmetric Influence of Attribute Displacement Performance on Customer Evaluation of Service Experiences: An Abstract
}

\author{
Liwu Hsu, Elten Briggs, and Timothy Landry
}

\begin{abstract}
This chapter examines how the displacement performance (i.e., a positive or negative change from the prior time period) of service quality attributes influences customers' service experiences. Random-effect GLS regression is applied to analyze this effect in a sample of over 50,000 observations from the post-transaction surveys of a Fortune 500 rental car company. Results confirm that displacement performance has a significant effect on customer experience evaluation, even after controlling for the influence of attribute positional performance (i.e., the static performance levels) and the customers' prior overall service experience evaluation. Further analyses indicate that the displacement performance effect is stronger for some service quality attributes when there is a short time interval between service deliveries. Finally, the study shows that negative displacement performance has a greater influence on experience evaluation than positive displacement performance. The results imply that managers should manage displacement perceptions in order to enhance customer experiences.
\end{abstract}

\footnotetext{
L. Hsu $(\bowtie) \bullet$ T. Landry

University of Alabama in Huntsville, Huntsville, AL, USA

e-mail: liwu.hsu@uah.edu; tim.landry@uah.edu

E. Briggs

University of Texas at Arlington, Arlington, TX, USA

e-mail: ebriggs@uta.edu
} 